\title{
METACOGNIÇÃO E ENSINO DE ENFERMAGEM: UMA COMBINAÇÃO POSSÍVEL?
}

Nilva Lúcia Rech Stedile ${ }^{1}$

Maria Romana Friendlander ${ }^{2}$

Stedile NLR, Friendlander MR. Metacognição e ensino de enfermagem: uma combinação possível? Rev Latino-am Enfermagem 2003 novembro-dezembro; 11(6):792-9.

Ensinar alunos a aprender a aprender é um desafio para as escolas que pretendem formar indivíduos autônomos e capazes de tomar decisões. Esses são aspectos essenciais para profissionais da área da saúde que se defrontam permanentemente com situações complexas e com uma multiplicidade de problemas a serem resolvidos pela atuação profissional. O desejo de mudanças, explícito no discurso dos educadores, não garante alterações substanciais na forma como o ensino vem sendo desenvolvido. Como promover essas alterações é uma pergunta ainda sem resposta. Os estudos desenvolvidos, nas duas últimas décadas, sobre metacognição parecem apontar uma estratégia possível de ser utilizada para transformar conhecimento em conduta profissional relevante, uma vez que favorece o pensar sobre o processo de pensamento e o desenvolvimento e controle de habilidades mentais capazes de promover maximização no uso das potencialidades individuais necessárias ao enfermeiro para resolver problemas de saúde.

DESCRITORES: aprendizagem; cognição; educação superior; enfermagem

\section{METACOGNITION AND NURSING TEACHING: A POSSIBLE COMBINATION?}

Teaching students to learn how to learn is a challenge for schools that intend to form autonomous people who are capable of taking decisions. These are essential aspects for health professionals who are permanently confronted with complex situations and a multiplicity of problems to be solved by professional actions. The desire for changes, which is explicit in educators' discourse, does not guarantee substantial changes in the form teaching is being developed. The question about how to promote these alterations still remains unanswered. Studies developed in the last two decades about cognition and metacognition seem to indicate a possible strategy to transform knowledge into relevant professional behavior, since it favors thinking about the thinking process as well as the development and control of mental abilities that are capable of maximizing the use of the individual potentialities necessary for a nurse to solve health problems.

DESCRIPTORS: learning; cognition; higher education; nursing

\section{METACOGNICIÓN Y ENSEÑANZA DE ENFERMERÍA: ¿UNA COMBINACIÓN POSIBLE?}

Enseñar al alumno para que aprenda, es un enorme desafío de las escuelas que intentan formar individuos con autonomía y con capacidad para tomar decisiones. Esta autonomía junto con la capacidad de tomar decisiones, son considerados aspectos primordiales para los profesionales del área de la salud, los cuales constantemente se enfrentan con situaciones complejas y con una diversidad de problemas para solucionarlos a través de su actuación profesional. El hecho de que esta intención esté involucrada en el discurso de los educadores, no garantiza que ocurran alteraciones importantes en la forma como la enseñanza está desarrollándose. ¿Cómo se pueden promover esas alteraciones? Esta es una pregunta aún sin respuesta. Los estudios desarrollados en las dos últimas décadas sobre cognición y metacognición al parecer se orientan hacia una estrategia posible de ser utilizada, para transformar el conocimiento en una conducta profesional relevante, ya que favorece el pensar sobre el proceso del pensamiento y el desarrollo y control de las habilidades mentales capaces de promover al máximo, el uso de las potencialidades individuales necesarias para que el enfermero pueda solucionar problemas de salud.

DESCRIPTORES: aprendizaje; cognición; educación superior; enfermería

\footnotetext{
${ }^{1}$ Enfermeira, Professor da Universidade de Caxias do Sul, Doutoranda, e-mail: nilvers@nsol.com.br; ${ }^{2}$ Enfermeira, Professor Titular, Orientador. Universidade Federal de São Paulo
} 
A Enfermagem, para redefinir seu compromisso frente às condições de saúde da população, precisa examinar, de forma rigorosa, a formação profissional oferecida aos graduandos nos cursos de Enfermagem. Atualmente, o ensino de enfermagem não só privilegia o estudo do corpo em sua dimensão biológica, como reforça a fragmentação desse corpo. Além disso, na maioria dos cursos, há uma grande preocupação em oferecer muita informação, em detrimento da formação e do desenvolvimento das capacidades de trabalhar com essa informação. Finalmente, o ensino de graduação atual não dá ênfase à formação do aprendiz autônomo que precisa conhecer sua maneira própria de apreender, para assumir a responsabilidade pela sua aprendizagem.

Parece que o desafio é desenvolver um ensino que possibilite ao aluno aprender a aprender. A metacognição, ao preocupar-se com a capacidade de o aluno pensar sobre o seu pensar e, dessa forma, desenvolver e controlar habilidades cognitivas, mostra-se um instrumento poderoso para aumentar a probabilidade de desenvolver a autonomia necessária aos futuros profissionais, para resolver problemas de saúde, normalmente complexos.

A relação entre a metacognição e a aprendizagem é difícil de ser explicada com precisão, em decorrência da complexidade dos conceitos envolvidos. Além disso, os conhecimentos produzidos sobre a cognição e seu controle são recentes e, conseqüentemente, a explicitação precisa da forma como o homem aprende ainda não foi possível. A possibilidade de estabelecer esta relação depende de esclarecimentos sobre, no mínimo, dois conceitos básicos: processo de aprendizagem e metacognição.

\section{APRENDER: FENÔMENO COMPLEXO E DIFÍCIL DE DEFINIR}

Com freqüência, o ensino em saúde ocorre de forma tradicional, num modelo "professor-centrado". O resultado dessa forma de organizar e propor ambientes de aprendizagem parece insuficiente para a formação de profissionais que necessitam muito mais do que reproduzir técnicas e conhecimentos propostos por autores consagrados, os quais, inúmeras vezes, são ineficazes em contextos cujas características sejam diferentes daquelas nas quais esses conceitos foram produzidos. A distância existente entre o que a sociedade precisa em termos de habilidades profissionais para melhorar a qualidade de vida e o que os cursos de formação têm oferecido, propiciou inúmeras críticas aos "modelos" de currículo existentes na área da saúde e o surgimento de novas teorias que serviram de base para tentativas de melhorar as aprendizagens dos alunos, em todos os níveis de formação.

Uma dessas abordagens, o Construtivismo, proposto a partir dos estudos de Piaget, tem como eixo teórico fundamental que o indivíduo é agente ativo no seu processo de conhecer, construindo significados, e definindo, além de seu próprio sentido, a representação da realidade existente, de acordo com suas experiências e vivências em diferentes contextos ${ }^{(1-2)}$. Essas representações podem mudar, e suas estruturas formam as bases sobre as quais são construídos ou reconstruídos os novos conhecimentos. De uma forma geral, o construtivismo estabelece o processo de aprendizagem como o desenvolvimento permanente e cada vez mais elevado da capacidade de elaboração própria ${ }^{(3)}$.

A idéia da aprendizagem reconstrutiva ${ }^{(4)}$ é marcada pela relação de sujeitos e tem, como objetivo principal, o desafio de aprender. Nesse ambiente, o professor assume a condição de "orientador maiêutico", que tem, como contexto central, a formação da competência humana, o processo do saber pensar, do aprender a aprender e um propósito ético-político.

O construtivismo, ao preocupar-se com o desenvolvimento do sujeito enquanto agente ativo no processo de aprender, é contrário ao pressuposto básico do ensino tradicional de que o conhecimento pode ser compreendido e compartilhado mediante transmissão de informações e de uma visão linear e simplificada dos fenômenos. Ao contrário, sustenta que o processo de formação tem como eixo fundamental a atividade intencional do aluno, na resolução de problemas do mundo real. "Cada indivíduo é sujeito da construção de seu próprio conhecimento, um processo individual e particular, só possível através da interação com o ambiente e com outros sujeitos e da formação de uma consciência reflexiva sobre sua aprendizagem"(5).

Podem ser destacados três aspectos básicos para uma abordagem construtivista de aprendizagem ${ }^{(6)}$ : 1) os conhecimentos são ativamente construídos ou reconstruídos (pelo aprendiz) em cada uma das situações onde estão sendo utilizados ou experimentados, e esta 
construção ocorre de forma individualizada, ou seja, depende da atividade mental de quem quer conhecer. Assim, os processos e os resultados gerados são diferentes de um indivíduo para outro e de um contexto para outro; 2) o "aluno" ocupa o centro do processo; o conhecimento é experimentado por meio de uma atividade cognitiva de criação de sentido pelo aprendiz ${ }^{(7)}$, e a aprendizagem acontece pela interação que o aprendiz estabelece entre os diversos componentes do seu meio ambiente, criando representações que são observáveis no discurso ou no comportamento e que são abertas a negociações e à reinterpretação; 3) o contexto de aprendizagem desempenha papel importante no seu grau e na sua intensidade, já que a atividade do aprendiz está inserida em um meio ambiente que aumenta ou diminui a possibilidade para estabelecimento de determinadas relações.

O conceito de ambiente de aprendizagem ${ }^{(2)}$ representa o lugar ou espaço onde ocorre a aprendizagem. Pressupõe a presença de atividades e recursos, pois o aluno utiliza ferramentas, coleta e interpreta informações, recebe orientação e suporte, e interage com outras pessoas. É um local onde os alunos podem explorar suas próprias metas de aprendizagem, exercendo autonomia e responsabilidade sobre a construção de seu próprio conhecimento; os alunos trabalham juntos em projetos e atividades, trocando suporte e aprendizagem entre si e com o ambiente.

Deslocar o foco principal do processo de ensino para a aprendizagem é uma tarefa difícil, por exigir uma alteração substancial na lógica tradicional de conduzir a aprendizagem. A mudança necessária significa substituir a certeza pela problemática da incerteza, o que valoriza o contexto do erro e da dúvida; a leitura linear dos fenômenos causa-efeito para uma leitura multideterminada; o trabalho fragmentado por cada especialidade do campo da saúde pela necessidade de interdisciplinaridade. De fato, as possibilidades de transformação dependem da contribuição das teorias provenientes de diferentes áreas do conhecimento e da capacidade do professor de demonstrar o quanto, e em que grau, cada área pode contribuir para solução de determinados problemas em saúde. Problemas esses que, na maioria das vezes, resultam da inter-relação entre inúmeras variáveis que o compõem e determinam.

Desenvolver habilidades profissionais complexas exige maximizar a utilização de habilidades cognitivas que possam auxiliar na reflexão sobre diferentes situações, de forma a analisar, examinar, criticar, sistematizar informações. Exige, também, que o aluno reflita permanentemente sobre seus próprios processos mentais, de forma a controlá-los gradativamente. Nesse ambiente flexível, o aluno interage de acordo com suas características, ritmo, interesse, e maximiza as estratégias mentais que sejam úteis à resolução de problemas ligados à saúde, de forma construtiva e colaborativa.

No caso, o que vai ser aprendido não são informações, teorias ou classificações existentes, mas o objeto de estudo são ações frente a situações existentes, com a qual o enfermeiro se defronta para gerar outra situação que seja desejável. O conhecimento deixa de ser finalidade do processo de ensino e transforma-se em instrumento a serviço do aluno, para ajudá-lo a identificar, com maior clareza, as variáveis presentes na situação, objeto de estudo, e as correspondentes e possíveis formas de controlar essas variáveis. Parece, também, ficarem mais nítidas as lacunas de conhecimento existentes. Conhecimentos ainda não produzidos são percebidos com maior facilidade em um processo de aprendizagem com essas características.

A capacidade de o profissional resolver problemas será provavelmente maior e mais rápida se o ensino possibilitar que o aluno desenvolva habilidades cognitivas que lhe permitam, gradualmente, tornar-se autônomo. Para entender melhor a relação entre o conceito e aprendizagem e autonomia, é importante que este último esteja claro. A autonomia $^{(8-10)}$ e seu desenvolvimento, não está relacionada ao isolamento; o surgimento do pensamento autônomo e lógico operatório é paralelo ao surgimento da capacidade de estabelecer relações cooperativas. Ser autônomo significa estar apto a construir cooperativamente o sistema de regras morais e operatórias necessárias à manutenção de relações permeadas pelo respeito mútuo. No campo da saúde, ser autônomo pode significar, muito mais, discutir os problemas e suas possibilidades de solução cooperativamente com os envolvidos, do que prescrever ações julgadas importantes do ponto de vista único do profissional.

Importante é perceber que há dois elementos fundamentais no processo de aprendizagem: o orientador e 0 aluno. A qualidade das aprendizagens depende das habilidades do orientador para construir ambientes que favoreçam as aprendizagens e do esforço reconstrutivo de cada aluno no processo. 
ENTENDENDO OS CONCEITOS DE COGNIÇÃO E METACOGNIÇÃO

Há muitos conhecimentos a serem construídos para o esclarecimento do que seja cognição e metacognição, sobre a relação entre estes dois conceitos e destes com o de inteligência. A ciência ainda não conhece o suficiente sobre o processo de construção do intelecto humano. Uma das razões possíveis é o fato de que a Psicologia também sofreu influência da visão cartesiana e mecanicista do mundo, do que decorreu uma separação entre mente/comportamento, que começou a ser questionada no final do século XIX. Como conseqüência, "algumas escolas psicológicas que conseguiram respostas mais consistentes a essas questões ainda são contemporâneas, e alguns de seus teóricos, das linhas congnitivistas-construtivista e humanistas, só na última década é que começaram a ser citados. Apesar dos conhecimentos produzidos em larga escala, nas últimas duas décadas, não há uma resposta simples e livre de contradição sobre como o homem aprende ${ }^{(11)}$.

Contribuir para construção de respostas sobre como o homem aprende passa, necessariamente, pelo estudo da cognição, considerando que as estruturas cognitivas formam as bases sobre as quais novos conhecimentos são construídos. Cabe destacar que a construção de significados na mente é um processo mental personalizado e individualizado, envolvendo diferentes habilidades cognitivas que, por sua vez, dependem da interação de fatores individuais e ambientais.

Os processos cognitivos são desenvolvidos na criança a partir das interações concretas que realiza, permitindo o fortalecimento de operações mentais, mediadas pelo pensamento, as quais, gradualmente, ampliam-se no aspecto da complexidade. Com o passar do tempo, não há mais necessidade do concreto, e as abstrações permitem ampliar, ainda mais, o desenvolvimento cognitivo. Os desdobramentos dessas habilidades cognitivas dependem, ainda, das interações subjetivas do próprio sujeito com o ambiente.

Considerando os seres humanos, há suficiente base factual, científica e filosófica para a suposição de que o homem desfrute de duas formas de consciência: 1) consciência imediata (absorção representativa, figurativa ou simbólica de nossas experiências, baseada na sensibilidade); 2) autoconsciência, que impõe ao sujeito um distanciamento da cognição ou de si mesmo, o que sugere o seu desdobramento em duas classes: a do objeto e a de si mesmo. A atenção, a seleção de objetos a perceber, pensar ou evocar, e a supressão intencional de conteúdos da consciência imediata, são elementos que ajudam a atuar sobre as atividades mentais ${ }^{(12)}$.

Outro aspecto a considerar é que nem sempre é fácil distinguir o cognitivo do metacognitivo. "Uma maneira de perceber a relação entre eles é entender que a cognição envolve-se com o fazer, enquanto que a metacognição tem a ver com escolher e planejar o que está sendo feito"(13).

Embora as pesquisas estejam bastante desenvolvidas em relação ao estudo da cognição, tais estudos parecem ainda não dar conta de esclarecer os variados e complexos mecanismos que integram e interagem na construção do conhecimento. Grande parte dos pesquisadores aceita a idéia de que o conceito de cognição inclui processos mentais superiores: conhecimento, inteligência, pensamento, imagem, criatividade, planejamento, geração de estratégias, raciocínio, classificação, inferência, conceituação, simbolização, linguagem, entre outros, os quais precisam ser muitas vezes associados para responder às exigências de um ambiente complexo. As teorias existentes não proporcionam isoladamente uma explicação satisfatória sobre como uma pessoa pensa e aprende, isto porque cada uma focaliza aspectos diferentes do desenvolvimento cognitivo e, em conjunto, podem dar respostas mais completas a essa questão.

Tendo presente os aspectos apresentados quanto ao conceito de cognição, é possível perguntar: em que medida esse conceito pode ajudar o aluno a aprender a aprender? Se a função da cognição é a adaptação e serve à organização do mundo que se experimenta ${ }^{(14)}$, utilizá-la permite ao indivíduo transformar em conceitos as experiências vividas. Por sua vez, os conceitos criados influenciam, de alguma forma e em algum grau, o comportamento dos indivíduos. Transformar conhecimento em conduta humana relevante depende da capacidade do indivíduo em construir conceitos e utilizálos no exercício profissional ${ }^{(15)}$. Dessa forma, o conhecimento é experimentado por meio de uma atividade cognitiva de criação de sentido pelo aprendiz ${ }^{(6-7)}$ e não transmitido. Nesse sentido, a percepção do indivíduo é importante para que ele possa construir representações mentais sobre os fenômenos. "A aprendizagem acontece pela interação que o aprendiz estabelece entre os diversos 
componentes do seu meio ambiente, que inclui as informações disponíveis (saberes científicos e saberes práticos). A natureza e o tipo de interações agilizadas dependem da percepção que o indivíduo tem dos diversos componentes"(6).

As representações são estruturas externas observáveis no discurso ou no comportamento e estão abertas a negociações ou reinterpretações ${ }^{(6)}$. Significa que o professor, ao observar tais comportamentos ou analisar o discurso próprio e dos alunos, pode utilizá-los como elementos de reflexão que, por sua vez, possibilitam a construção de novos conceitos e representações.

Numa dada situação, o indivíduo resgata certo número de elementos que Ihe permitem construir uma representação daquela situação a ser gravada cognitivamente na memória. Essas representações podem servir para inferir aspectos de novas situações encontradas ou simular situações ${ }^{(16)}$. A aprendizagem realiza-se pela construção de modelos mentais e pela sua negociação; suas propriedades são verificadas ou corrigidas por meio da simulação ou interação social.

\section{ASPECTOS ESSENCIAIS PARA COMPOR UM CONCEITO DE METACOGNIÇÃO}

Essencialmente, a metacognição refere-se ao conhecimento dos processos de cognição e seus produtos. Engloba atividades de monitoramento e conseqüentes regulações e instrumentalizações desses processos, em relação a objetivos ou dados cognitivos. Dessa forma, está ligada às estratégias utilizadas pelos indivíduos, as quais monitoram, testam, ordenam e controlam as habilidades cognitivas, nos esforços individuais para aprender.

Estudos sobre metacognição ${ }^{(17-19)}$ têm distinguido dois componentes maiores desse fenômeno: 1) conhecimento sobre a cognição e 2) regulação da cognição. O conhecimento sobre a cognição ${ }^{(20)}$ inclui três subprocessos que facilitam o aspecto reflexivo da metacognição: conhecimento declarativo (autoconhecimento e conhecimento de estratégias); conhecimento processual (conhecimento sobre como usar estratégias); conhecimento condicional (conhecimento sobre quando e por que usar estratégias). A regulação da cognição, por sua vez, inclui um número de subprocessos que facilitam o aspecto de controle das estratégias de pensamento (planejamento, gerenciamento de informações, monitoramento da compreensão, depuração, avaliação).

O termo metacognição refere-se ao conhecimento sobre o processo de cognição de um modo geral, bem como o reconhecimento e a identificação de processos cognitivos próprios. Em um nível geral, envolve conhecimento sobre, entendimento de, e acesso à cognição e a recursos cognitivos $^{(21)}$. É definida como o conhecimento e o controle que o indivíduo tem de seus próprios pensamentos e atividades de aprendizagem e tem sido relacionada com aptidões cognitivas, tais como, inteligência, leitura e memória ${ }^{(22)}$.

Pode também ser definida como um conjunto de conhecimentos e estratégias de alto nível que guiam e regulam a atividade cognitiva e, conseqüentemente, a performance ${ }^{(23)}$. A avaliação desse processo e a identificação dos meios para melhorá-lo é um desafio para a aprendizagem, retenção de informações e capacidade de resolver problemas. É também uma forma de remediar ${ }^{(21)}$ o ensino.

Combinar a resolução de problemas com trabalho de grupo, comunicação, reflexão e métodos alternativos de avaliação em situações de ensino, exige uma série de orientações $^{(24)}$. Tais orientações podem ser também utilizadas para resolução de problemas no campo da saúde. Cada indivíduo deve pensar sobre si mesmo, nos seguintes aspectos: a) pensar sobre o próprio processo de pensamento durante a resolução de problemas; b) pensar sobre potencialidades e limitações; $c$ ) pensar sobre o próprio conhecimento; d) pensar sobre crenças e concepções próprias; e) pensar sobre próprias atitudes; f) pensar sobre a influência que crenças, concepções e atitudes podem ter sobre o fazer profissional; g) pensar sobre a motivação para aprender e para superar dificuldades de aprendizagem; h) pensar sobre o monitoramento e controle de seu próprio esforço para resolver problemas relacionados às situações profissionais.

A análise dessas orientações permite afirmar que a metacognição desenvolve-se a partir da capacidade de o homem refletir sobre si mesmo, sobre seu fazer, sobre os processos mentais que facilitam esse fazer e sobre as estratégias que utiliza para resolver problemas com os quais se defronta pessoal e profissionalmente.

Quando o indivíduo aprende a monitorar sua dinâmica cognitiva, parece poder produzir um duplo efeito: apreensão metacognitiva de conteúdos e aspectos 
operacionais da mente e autoconsciência do papel profissional, como pessoa dotada de valores, motivos, disposições afetivas, limitações e tendências comportamentais. Resumidamente, refere-se à "cognição sobre a cognição" e ao "controle executivo". O treinamento em habilidades de controle executivo, por sua vez, favorece um comportamento mais reflexivo, o que conduz a facilitar a aquisição e transferência de estratégias mais adequadas para a aprendizagem.

\section{A UTILIZAÇÃO DA METACOGNIÇÃO EM SALA DE AULA E SUA RELAÇÃO COM O APRENDIZADO}

A utilização da metacognição como ferramenta para a aprendizagem pressupõe o desenvolvimento gradual da capacidade de o aluno identificar, controlar e desenvolver seus processos cognitivos e utilizá-los na resolução de problemas relacionados à saúde. Desenvolver a metacognição permite a identificação, monitoramento, auto-regulagem e orquestração das habilidades mentais pelos próprios alunos.

A habilidade metacognitiva está presente em todos os indivíduos em níveis diferenciados de compreensão e intensidade de expressão ${ }^{(25)}$. Identificar esses níveis e favorecer o processo de tomada de consciência do aluno em relação às formas como aprende, parece ser, ao mesmo tempo, um desafio para a educação superior e um requisito para alterar a forma tradicional de organização do ensino. A progressiva tomada de consciência por parte do indivíduo, de seus próprios potenciais, deficiências e conhecimentos, leva ao melhor domínio de suas atividades e a um progressivo ganho de produção ${ }^{(26)}$.

Parte da aprendizagem de um bom estudante pode ser sua capacidade de concentrar atenção para sua própria mente e para seus graus de entendimento. $\mathrm{O}$ bom estudante pode ser o que diz o que não entendeu, simplesmente porque mantém uma checagem constante de seu entendimento. $\mathrm{O}$ estudante com grau maior de dificuldades de aprendizagem, a maior parte do tempo, não sabe se entendeu ou não. Assim, o problema não é com o estudante que pergunta o que não sabe, mas é fazer com que atente para a diferença entre o que sabe e o que não sabe. De uma forma geral, os estudantes que desenvolvem habilidades metacognitivas estão mais aptos a mudarem seus próprios hábitos de estudo e seu aprendizado de estratégias, quando se deparam com uma tarefa nova ${ }^{(13)}$.

Os componentes metacognitivos podem ser desenvolvidos ou reconfigurados em sala de aula, desde que o ambiente seja favorável a que o aluno reconheça e utilize características cognitivas motivacionais, pessoais e situacionais; que eles estejam permanentemente engajados na avaliação e administração de suas próprias aprendizagens; que sejam estimulados a pensar sobre processos e não sobre conteúdos; que sejam motivados e valorizados na atividade de descrever os processos envolvidos e as estratégias cognitivas utilizadas na construção de significados e na resolução de problemas; que transformem em palavras as imagens mentais produzidas; que avaliem a qualidade das estratégias de pensamento. É necessário que o ensino preocupe-se menos em instruir ou induzir as pessoas a desempenharem rotinas de processamento específicas (por exemplo, memorizar a disposição do material em uma mesa de instrumental cirúrgico) e mais a compreender o significado de tais atividades. Nessa situação, a transferência de habilidades cognitivas para novas situações é mais provável.

Um dos aspectos centrais da utilização da metacognição como facilitadora de aprendizagens é a seleção de estratégias, seu uso e a transferência para novas situações. Uma estratégia inclui o conhecimento dos processos usados voluntariamente para o alcance de determinado fim cognitivo. Desenvolvemos ações ou estratégias cognitivas para alcançar e monitorar progresso $\operatorname{cognitivo}^{(13)}$. As estratégias são compostas por operações cognitivas que são conseqüência natural da execução de tarefas e podem ser vistas como um conjunto mental de operações interdependentes que podem ser modificadas em resposta a diferentes situações. Essas estratégias e seus desempenhos correspondentes podem ser controlados.

As estratégias conseguem executar os propósitos cognitivos e são potencialmente conscientes e controláveis. Sua função é ajudar o aprendiz a executar operações essencialmente cognitivas que produzem "insights" eficientes no processo de aprendizagem. Se uma das capacidades da inteligência é auto regular a aprendizagem, então, significa planejar estratégias que vão ser usadas em cada situação de aprendizagem, aplicar e controlar o processo de utilização de cada uma delas, avaliar o processo para detectar erros e modificar a 
estratégia, tendo como conseqüência uma nova atuação.

De posse dessas informações, o orientador de aprendizagens pode facilitar a reflexão sobre as estratégias usadas pelos alunos dos cursos de Enfermagem para resolver os problemas relacionados à saúde.

No caso do uso da metacognição para resolução de problemas, ao aluno cabe ${ }^{(27-28)}$ :

1. reconhecer que há um problema, caracterizá-lo e reconhecer que há necessidade de intervenção (analisar e caracterizar o problema);

2. usar raciocínio inferencial para acessar as probabilidades de hipóteses, explicitando as informações que eles já possuem (refletir sobre o que sabe e o que não sabe quanto à solução);

3. elaborar um plano para resolução do problema;

4. prognosticar o resultado de suas tentativas no uso de estratégias;

5. prognosticar as dificuldades na tarefa;

6. planejar estratégias de estudo;

7. monitorar as tentativas para aprender e tentar de outras formas (checar ou monitorar o processo);

8. questionar sobre habilidades próprias que capacitariam em determinadas inadequações.

Ao agir levando em conta esses aspectos, o aluno progride no controle consciente dos processos cognitivos,

\section{REFERÊNCIAS BIBLIOGRÁFICAS}

1. Duffy TM, Jonassen DH. Construtivism: new implications for instructional technology. Constructivism and the technology of instruction: a conversation. Hillsdale (NJ): Lawrence Erlbaum; 1992.

2. Wilson BG. Metaphors for instruction: why we talk about learning environments. Educ Technol 1995; 25-30.

3. Grossi EPM, Bordin J, organizadores. Construtivismo póspiagetiano: um novo paradigma de aprendizagem. Petrópolis (RJ): Vozes; 1993.

4. Demo P. Professor e Teleducação. Tecnol Educ 1998 out/ nov/dez; 26(143):52-64.

5. Struchiner M, Rezende F, Ricciardi RMV, Carvalho MAP. Elementos fundamentais para o desenvolvimento de ambientes construtivistas de aprendizagem. Tecnol Educ 1998; 26(142):3-11.

6. Deschênes AJ, Bilodeau H, Bourdages L, Dionne M, Gagné $\mathrm{P}$, Lebel C, Rada-Donath A. Construtivismo e formação à distância. Tecnol Educ 1998; 26(140):3-10.

7. Jonassen DH, Campbell J, Davidson ME. Learning with media: restructuring the debate. Educ Technol Res Dev 1994; 42:31-9.

8. Piaget J. A psicologia da inteligência. $2^{a}$ ed. Lisboa: Fundo de Cultura; 1967.

9. Piaget J. A equilibração das estruturas cognitivas: problema central do desenvolvimento. Rio de Janeiro (RJ): Zahar; 1976. 10. Piaget JA. Para onde vai a educação? Rio de Janeiro (RJ): José Olímpio; 1984 de forma que possa gradativamente maximizar o uso de suas habilidades cognitivas na resolução de problemas e identificar dificuldades cognitivas para utilizar estratégias que desenvolvam tais habilidades.

Cabe ressaltar que as tarefas/problemas que são oferecidos aos alunos devem ser dotados de importância para que eles mantenham motivação e interesse durante a sua realização. É importante, nesse sentido, que o aluno tenha claro conhecimento sobre o propósito das aprendizagens para a vida profissional e em que medida as generalizações das estratégias beneficiarão sua vida cotidiana. O grau de envolvimento do aluno com a tarefa proposta é um fator importante na diminuição de falhas de monitoramento dos processos cognitivos, ocasionadas, principalmente, por falta de concentração e atenção.

Pressupor que os estudos sobre metacognição possam, por si só, resolver os problemas de aprendizagens, especialmente complexas, como as necessárias para atuar em saúde, é, no mínimo, ingenuidade intelectual. No entanto, estudá-la e utilizá-la como instrumento potencializador de resultados no ensino parece ser um caminho importante a ser considerado pelos profissionais que acreditam que é possível construir um novo modelo de currículo em Enfermagem e, conseqüentemente, consolidar um novo perfil profissional.

11. Ramos EMF. Análise ergonômica do sistema hiper Net buscando o aprendizado da cooperação e da autonomia. [Tese]. Florianópolis (SC): Universidade Federal de Santa Catarina; 1996.

12. Krüger H. Informática educativa e metacognição. In: Conferência Internacional de Logo; 1992. Petrópolis; 1992.

13. Messer C. Processos Metacognitivos no ensino de conteúdos: monitoramento cognitivo de professores em séries iniciais. [Dissertação]. Rio Grande do Sul (RS): PUC/ RS; 1995.

14. Jegede $O$. A constructivist epistemology and its implications for contemporary research in distance learning. In: Evans T, Juler P, editors. Research in distance education. Deakin: Institute of Distant Education; 1992. p.21-9.

15. Botomé SP. O enfermeiro como profissional de nível superior. In: Semana Interinstitucional de Enfermagem; 1995; Caxias do Sul. Anais. Caxias do Sul: Universidade de Caxias do Sul; 1995.

16. Greeno JG. Environments for situated conceptual learning. In: Birnbaum L, editor. International Conference on Learning Sciences; 1991. Charlottsville: Association of the Advancement of Computing Education; 1991. p.211-9.

17. Brown AL. Metacognition, executive control, self-regulatiion and other more mysterious mech-anisms. In: Weinert FE, Kluwe $\mathrm{RH}$, editors. Metacognition, motivation and understanding. Hillsdale (NJ): Lawrence Erlbaun; 1987.

18. Flavell JH. Speculation about the nature and development of metacognition. In: Weinert F, Klume R, editors. Metacognition, motivation and understanding. Hillsdale (NJ): Lawrence Erlbaum; 1987. p.21-9. 
19. Jacobs JE, Paris SG. Children's metacognition about reading: issues in definition, measurement, and instruction. Educ Psychol 1987; 22:255-78.

20. Schraw G, Dennison RS. Assessing Metacognitive Awareness. Contemmporary- Educ Psychol 1994; 19:46075.

21. Minnaert A, Janssen PJ. The additive effect of regulatory activities on top of intelligence in relation to academic performance in higher education. Learning and Instruction. J Eur Assoc Res Lear Instruc 1999 Feb; 9(1):77-91.

22. Swanson HL. Influence of Metacognitive Knowlwdge end Aptitude on Problem Solving. J Educ Psychol 1990; 82(2):30614.

23. Huet N, Mariné C. Techniques D"évaluation de Ls Métacognition. I Les Mesures Indépendantes de L"exécution de Tâches. L'Année Psychol 1998; 98:711-26.

24. Carpenter TP. Teaching as problem solving. In: Charles RI, Silva EA, editors. Research agenda for mathematics education the teaching and assessing of mathematical problem solving. Reston (VA): National Council of Teachers of Mathematics; 1998. p.187-202.

25. Mettrau MB, Mathias MT. O papel social da prática pedagógica do professor na promoção das capacidades sócio-cognitivo-afetivas do aluno. Tecnol Educ 1998; 26:306.

26. Wood D. Como as crianças pensam e aprendem. São Paulo: Martins Fontes; 1996.

27. Brown AL. The development of memory: knowing, knowing about knowing, and knowing how to know. Adv Child Dev Behav 1975; 10:103-52.

28. Brown AL. Knowing when, where and how to remember: a problem of metacognition. In: Glaser R, editor. Advances in instructional psychology. Hillsdale (N.J): Lawrense Erlbaum Associates; 1978. 